\title{
Editorial \\ Special Issue on Sensors Systems for Structural Health Monitoring
}

A LMOST a dozen people lost their lives when the eight lane I-35 Bridge collapsed into the Mississippi River at rush hour during August 2007. After that there were several other incidents of bridge collapse that took place in China, India, and Pakistan. Recently, a high-rise building in Cologne, Germany, collapsed with little apparent warning.

Given such incidents it has become of increasingly paramount importance to develop methods detecting the degradation or damage that result in these events. Thus, buildings and critical infrastructure could be monitored, much like a patient in a hospital, for signs of degradation or impending disability or collapse. From which arises the name Structural Health Monitoring or SHM.

Early detection of damage from natural hazards or other events requires intelligent methods and sensors that are able to take a potentially diverse array of data and create a picture of the structure's condition and state. Thus, the sensors must have access to or contain intelligent features to detect the problem. It was therefore expected that a Special Issue on Sensors and Systems for Structural Health Monitoring would provide many new ideas for the detection and inspection of structures to increase their safety and reliability.

Hence, there is an urgent need to design, develop, fabricate of different types of sensors and sensing technology, as well as the associated methods or computation. The overall goal is the creation of noninvasive systems and techniques to determine the integrity of a material, component or structure, to quantitatively measure some characteristics of the system's level of damage (or health) to predict and thus avert catastrophic failure. Currently, few such complete systems exist. Although necessary technology components are well reported, there has been relatively lesser advance in implementing these systems in full.

It should be noted that this task is actually quite daunting, as current structural design methods and codes often consider a great deal of over design for safety. This makes detecting moderate to modest damage and determining the resulting implications for the potential safety difficult. In short, the signal-tonoise ratio of this problem is often not good.

So, in short, the fabricated sensor systems should be able to inspect or measure without doing any harm or damage of the system. They should also be robust to poor signal-to-noise ratio compared to the level of damage they are trying to detect in these critical infrastructure. Finally, they need to be highly reliable

Digital Object Identifier 10.1109/JSEN.2009.2028173 and operate without input for long periods of time, potentially over years.

This Special Issue has focused on the different aspects of sensing technology, i.e., high reliability, adaptability, recalibration, information processing, data fusion, validation, and integration of novel and high performance sensors specifically aims to use to inspect mechanical health of structure and similar applications. While future interest in this field is ensured by the constant supply of emerging modalities, techniques and engineering solutions, as well as an increasing need from aging structures, many of the basic concepts and strategies have already matured and now offer opportunities to build upon.

After the review, a total of 34 high-quality papers have been accepted for this Special Issue.

We are very happy to be able to offer the readers of the IEEE SENSORS JOURNAL such a diverse Special Issue both in terms of its topical coverage and geographic representation. We do hope that the journal readers would find it interesting, thought provocative, and useful in their research and practical engineering work.

We would like to present our whole-hearted thanks to all the authors who have contributed their work to this Special Issue. We are also sincerely grateful to the excellent team of reviewers from all around the world who have generously devoted their precious time to review the submitted manuscripts. Finally, we are grateful to Eileen Murray and her staff at the IEEE for the extremely valuable editorial support and production of this Special Issue.

SUBHAS CHANDRA MUKHOPADHYAY, Guest Editor
Massey University
School of Engineering and Advanced Technology
Palmerston North 5301, New Zealand
s.c.mukhopadhyay @ massey.ac.nz

J. GeOFFrey CHASE, Guest Editor University of Canterbury Department of Mechanical Engineering Christchurch 8140, New Zealand geoff.chase@canterbury.ac.nz

NORBERT MEYENDORF, Guest Editor University of Dayton Research Institute Center for Materials Diagnostics Dayton, OH 45469-0101 USA norbert.meyendorf@izfp-d.fraunhofer.de 


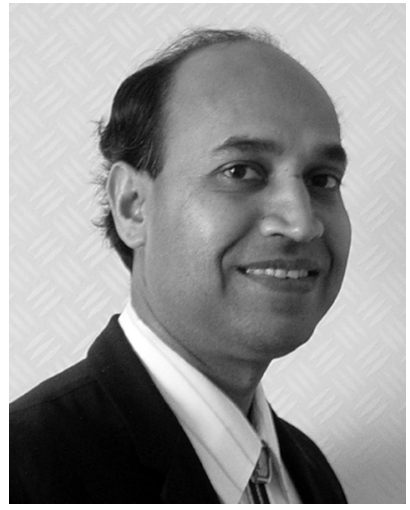

Subhas Chandra Mukhopadhyay (M'97-SM'02) was born in Calcutta, India. He graduated from the Department of Electrical Engineering, Jadavpur University, Calcutta, India, in 1987 with a Gold Medal and received the M.S. degree in electrical engineering from the Indian Institute of Science, Bangalore, in 1989, the Ph.D. degree in engineering from Jadavpur University, in 1994, and the Doctor of Engineering degree from Kanazawa University, Kanazawa, Japan, in 2000.

From 1989 to 1990, he worked almost two years in the Research and Development Department of Crompton Greaves, Ltd., India. In 1990, he joined as a Lecturer in the Department of Electrical Engineering, Jadavpur University, and was promoted to Senior Lecturer of the same department in 1995. Obtaining Monbusho Fellowship, he went to Japan in 1995. He worked with Kanazawa University, as a Researcher and Assistant Professor until September 2000. In September 2000, he joined the Institute of Information Sciences and Technology, Massey University, New Zealand, as a Senior Lecturer. Currently, he is working as an Associate Professor with the School of Engineering and Advanced Technology, Massey University. He has authored over 200 papers in different international journals and conferences, coauthored a book and wrote a book chapter and edited eight conference proceedings. He has been a guest editor of three international journal special issues. His fields of interest include smart sensors and sensing technology, electromagnetics, control, electrical machines, and numerical field calculation, etc.

Dr. Mukhopadhyay is a Fellow of the IEE (U.K.), an Associate Editor of the IEEE SEnsors Journal. He is also an Associate Editor for the IEEE TRANSACTIONS ON INSTRUMENTATION AND MEASUREMENTS. He is on the Editorial Board of the e-Journal on Non-Destructive Testing, Sensors and Transducers, Transactions on Systems, Signals and Devices (TSSD). He is on the Technical Program Committee of the IEEE Sensors Conference, the IEEE IMTC Conference, and the IEEE DELTA Conference. He was the Technical Program Chair of ICARA 2004, ICARA 2006, and ICARA 2009. He was the General Chair of ICST 2005, ICST 2007, and ICST 2008. He was the General Co-Chair of the IEEE Sensors Conference 2008. He is currently organizing the IEEE Sensors Conference 2009 as the General Chair to be held during October 25-28, 2009, Christchurch, New Zealand.

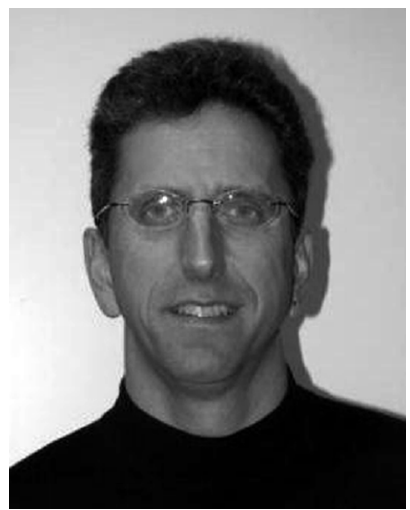

J. Geoffrey Chase received the B.S. degree in mechanical engineering from Case Western Reserve University, Cleveland, OH, in 1986, and the M.S. and Ph.D. degrees from the Departments of Mechanical and Civil Engineering, Stanford University, Stanford, CA, in 1991 and 1996, respectively.

He spent six years working for General Motors between various degrees and a further five years consulting and doing research in Silicon Valley, including positions at Xerox PARC, GN ReSound, and Infineon Technologies AG, before taking his current position at the University of Canterbury, Christchurch, New Zealand, in 2000. His fundamental research interests include automatic control, structural dynamics and vibrations, dynamic and systems modeling. These areas have been primarily applied in his research to biomedical and clinical medical devices with a focus on creating efficient delivery and optimized, patient-specific health care delivery. Specific accomplishments include the development of two startup companies focusing on novel breast cancer screening technologies, and model-based therapeutics in critical care medicine. He has published over 390 international, refereed journal and conference papers in these areas over the last eight years, and is an inventor on over eight U.S. and European patents with 5-10 more in process. He is on the editorial board of three journals in bio-engineering and clinical medicine, and is a member of the International Federation on Automatic Control (IFAC) Technical Committee (IFAC TC 8.2) on Biological and Medical Systems.

Dr. Chases's research team has also won three NZ Young Scientist of the Year Awards in the last five years, and won the IPENZ Design Award and NZ Health Innovation Supreme Award in 2005-2006. 


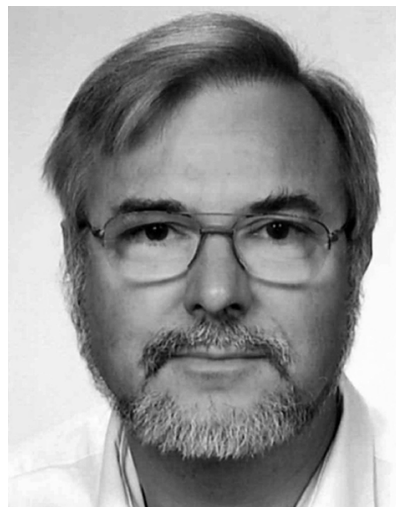

Norbert Meyendorf was born in Germany in 1953. He graduated with a degree in physics and received the Ph.D. degree in solid-state physics form the Martin Luther University, Halle, Germany, in 1980, and the Science Degree (habitation) in welding technology and martial science from the Technical University Magdeburg, in 1986.

From 1987 to 1992, he was an Assistant Professor at the Technical University Magdeburg, Magdeburg, Germany. From 1979 to 1987, he developed reaction kinetic models for metallurgical process in arc welding at the Central Welding Institute (ZIS). From 1992 to 2000, he was Head of the Department of Materials Characterization, Fraunhofer Institute for Nondestructive Testing, Saarbrücken, Germany. In 2000, he moved to Dayton, OH, where he took over the responsibility for the Center for Materials Diagnostics at the University of Dayton Research Institute. Since 2003, he has been on the Graduate Faculty at the University of Dayton. He shares the teaching activity at Dayton University with the position of Deputy Director of the Fraunhofer Institute for Nondestructive Testing and in this position is responsible for the Institutes Branch in Dresden (IZFP-D), Germany. He introduced Sensor Technology and Structural Health Monitoring into the research portfolio of IZFP-D that in recent years became a leading position in this area in Germany. He is author or coauthor of more than 200 original papers and editor of several books and conference proceedings. Since 2007, he has been Guest Professor of Micro and Nano Testing Methods at the University of Technology, Dresden. He is member of the Advisory Board of the Center for Micro- and Nano-Materials, Berlin, Germany, and an NSF-funded five-year PIRE-ISHM program "Intelligent Structural Health Management of Safety-Critical Aerospace, Mechanical, and Civil Structures."

Dr. Meyendorf is Vice President of the European Center for Micro-and Nano Reliability (EUCEMAN), Chair of the Technical Committee for Structural Health Monitoring of the Germans NDE Society (DGZfP), and Member of the Technical Committees "Aerospace" and "Materials Characterization." He served as Chair or Co-Chair of the SPIE Symposia "Smart Structures and NDE." 